Dieses Dokument ist eine Zweitveröffentlichung (Postprint Version) / This is a self-archiving document (accepted version):

Adrian Ehrenhofer, Thomas Wallmersperger

Adjustable fluid and particle permeation through hydrogel composite membranes

Erstveröffentlichung in / First published in:

Journal of Intelligent Material Systems and Structures.2018, 29 (3), S. 310 - 322. Sage Journals. ISSN 1530-8138.

DOI: https://doi.org/10.1177/1045389X17704065

Diese Version ist verfügbar / This version is available on:

https://nbn-resolving.org/urn:nbn:de:bsz:14-qucosa2-742319 


\title{
Adjustable fluid and particle permeation through hydrogel composite membranes
}

\author{
Adrian Ehrenhofer and Thomas Wallmersperger
}

\begin{abstract}
Membranes act as smart structures in respect to their permeation abilities. Control of particle and fluid permeation through a synthetic membrane can be achieved by using different effects like size-exclusion or electromagnetic interactions that occur between the particles and membrane pores. The simulation of controlled permeability provides an insight into the smart behavior of membranes for chemical signal processing, sensing interfaces or lab-on-a-chip devices. In the current work, we model the underlying physical processes on a microfluidic level using the engineer's approach of laminar flow through pipes. Different pore geometries inside a composite membrane system consisting of a polyethylene terephthalate support membrane and a poly( $\mathrm{N}$-isopropylacrylamide) hydrogel-layer are investigated. Simulations for different states of thermally induced pore opening are performed for free and blocked states. From the results we derive paradigms for the design of a membrane system for microfluidic cell-size profiling considering stimulus-range, pore shape and measurement setup.
\end{abstract}

\section{Keywords}

Polymeric membranes, finite element simulation, hydrogel swelling, microfluidics, micromechanical pore valve, particle separation

\section{Introduction to membranes, selectivity and permeation control}

Biological or synthetic membranes are barrier structures with a certain permeation performance. While biological membranes are self-assembling bilayers of amphiphilic phospholipids (Sarles and Leo, 2010; Singer and Nicolson, 1972), synthetic membranes can be composed of any porous material. In technology, mostly polymeric membranes are used for filtration processes like reverse osmosis and ultra-filtration (Ulbricht, 2006). In biology, the signaling and information processing abilities are of primary importance.

The key feature for membrane performance is selectivity, that is, the ability of membranes to allow specific particles to pass and to block others. In nature, selectivity is realized by specific binding reactions in channels, that is, biological permeation structures with selectivity. They consist of proteins, which are embedded in the membrane (Gouaux and MacKinnon, 2005).

To imitate selectivity, channel proteins can be inserted into artificial membranes that, in return, must allow their functioning (Hirano-Iwata et al., 2008). Non-selective channels that can be mechanically activated were implemented by Najem et al. (2015). For non-bilayer-lipid-membranes, the technological counterpart of selectivity can be size-exclusion of particles or electrostatic exclusion of ions (Daiguji, 2010), see Figure 1.

\section{Gating and permeation control}

Transport through biological membranes can be controlled by gating (DeCoursey and Hosler, 2014). Different stimuli allow a conformational change in the structure of a channel protein, changing the permeability-state from open to closed as presented, for example, by Martinac et al. (2008) (see Figure 2).

The technological equivalent to gating in natural membrane transport is permeation control in microfluidic or nanofluidic channels through electrostatic

\footnotetext{
Technische Universität Dresden, Institut für Festkörpermechanik, Germany

Corresponding author:

Adrian Ehrenhofer, Technische Uniersität Dresden, Fakultät

Maschinenwesen, Institut für Festkörpermechanik, George-Bähr-Str. 3c,

Dresden, Germany.

Email: adrian.ehrenhofer@tu-dresden.de
} 


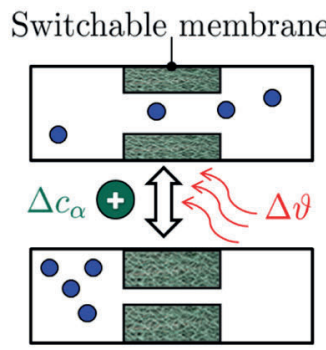

(a)

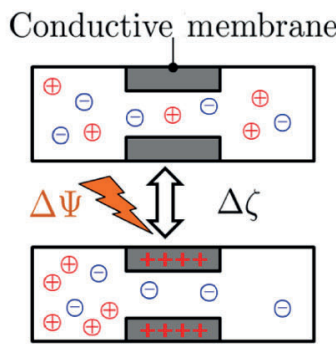

(b)

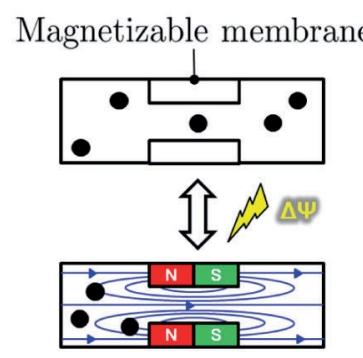

(c)

Figure I. (a) Size-exclusion and (b) electrostatic exclusion are the technological counterparts to biological selectivity. They can be manipulated in polymeric membranes to mimic gating. (c) Magnetic exclusion due to inhomogeneous magnetic fields does not occur in nature, but can be realized with magnetizable membranes.

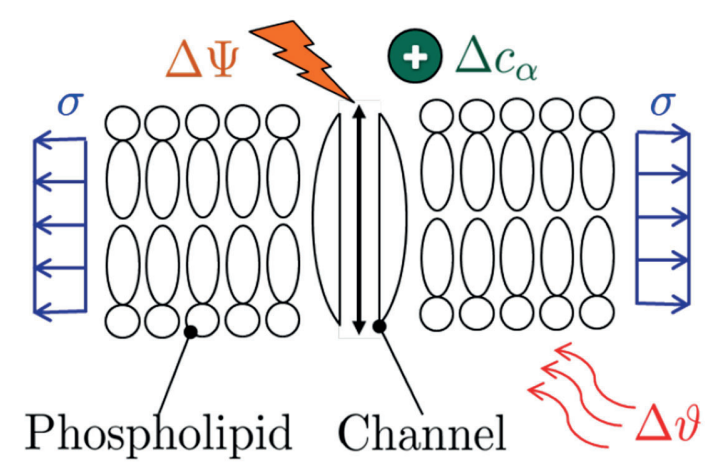

Figure 2. Gating in different cell channels occurs due to the associated stimulus like a voltage $U_{\text {Membrane }}=\Delta \Psi$ in a voltagegated potassium channel $\mathrm{K}_{V}$ or by a temperature difference $\Delta \vartheta$ in a temperature-sensitive cation channel TRPVI.

exclusion or size-exclusion (Daiguji, 2010). While natural gating is based on the complex structure of channel proteins inside phospholipid bilayers, the technological principles are much simpler. The size-exclusion is based on the fact that a rigid sphere of diameter $d_{\text {Sphere }}$ cannot pass through a pore in a membrane with diameter $d_{\text {Pore }}$ that is roughly equivalent or lower. Electrostatic exclusion (also called Donnan-exclusion) is due to repellent forces exerted on ions or charged particles in proximity to charged surfaces. The permeation through synthetic membrane pores can hence be controlled by pore geometry variation, see Figure 1(a), or through the application of surface charges, see Figure 1(b). In an inhomogeneous magnetic field, forces on ferromagnetic particles can be observed. This could also be used to achieve magnetic exclusion (Figure 1(c)).

Different groups work on the realization of sizeexclusion using porous polymer membranes (Adrus and Ulbricht, 2012; Darvishmanesh et al., 2015; Frost and Ulbricht, 2013; Ito et al., 1997; Park et al., 1998). In these approaches, the membrane changes its porosity due to an external stimulus. Nguyen et al. (2015) use special channel geometries to achieve cell separation, while Otto et al. (2015) sort cells on the basis of their deformability. A closer look at the fluid streaming through biological channels using the continuum theory is taken for example, by Tu et al. (2013), Zheng et al. (2011) and Chen et al. (1997). A continuum theory is also used for artificial pores (Cervera et al., 2005). Comparisons to theories on the molecular level have shown that the limits of continuum theory lie at a smaller level (Corry et al., 2000a,b). The behavior of hydrogels for permeation control in microfluidic channels through valves is investigated, for example, by Arndt et al. (2000) and Richter et al. (2003). An overview denoting different alternatives to fluidic separation of biological cells as envisaged by our membrane system is shown in Shields et al. (2015).

\section{Description of the switchable membrane}

The present article deals with hydrogel layered patterned membranes that allow selective blocking of particles with a defined diameter as presented in our previous work (Ehrenhofer et al., 2016a). This is achieved through control of the pore shape by reversible swelling of the hydrogel layer. The underlying model for the material behavior of the hydrogel constituent in the composite membrane is depicted here because it forms the basis of the following investigations of flow through the switching membrane.

Material behavior. Hydrogels like poly(N-isopropylacrylamide) (PNIPAAm) perform a reversible swelling due to hydrophilic interactions between the polymer side groups and surrounding water molecules. At temperatures higher than the lower critical solution temperature (LCST), the screening properties of water diminish and the attracting interactions of the polymer lead to the observed deswelling (Gerlach and Arndt, 2009). Various hydrogels with different stimuli responsiveness can be synthesized. When they are surface polymerized onto a backbone membrane, their swelling behavior is 

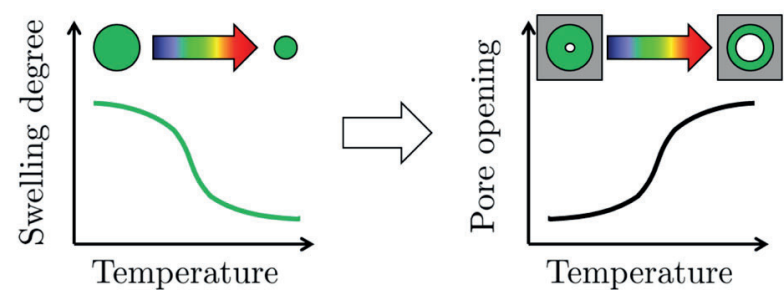

Figure 3. Due to the clamped configuration, a deswelling of the hydrogel at higher temperatures (left) leads to an opening of the pore structure (right).

inverted to a pore opening over the stimulus, see Figure 3.

A model based on the thermal expansion to describe the hydrogel swelling was derived and successfully compared to experimental studies of the temperaturedependent opening and closing of the pores (Ehrenhofer et al., 2016a; Keller et al., 2011). The underlying balance equations, kinematics and material law are

$$
\begin{gathered}
\text { Balance equations : } \sigma_{k l, k}+f_{l}=0 \quad \sigma_{k l}=\sigma_{l k} \\
\text { Kinematic relations : } \varepsilon_{k l}=\frac{1}{2}\left(u_{k, l}+u_{l, k}\right) \\
\text { Constitutive equations : } \sigma_{k l}=E_{k l m n}\left(\varepsilon_{m n}-\alpha(\vartheta) \delta_{m n} \Delta \vartheta\right)
\end{gathered}
$$

The stresses are $\sigma_{k l}$ in tensor notation with $k, l \in[x, y, z]$ and the spatial derivatives are denoted as ()$_{, k}=\partial / \partial x_{k}$. Volume loads $f_{l}$ are not considered in the model. For the kinematic relations (equation (2)) with the displacements $u_{k}$, small displacement gradients are supposed. The total strain is composed of elastic and thermal strains $\varepsilon_{k l}=\varepsilon_{k l}^{\mathrm{th}}+\varepsilon_{k l}^{\mathrm{el}}$, where the thermal strain is gained through the temperature dependent thermal expansion coefficient $\alpha(\vartheta)$ and the difference temperature to a reference state $\Delta \vartheta=\vartheta-\vartheta_{\text {ref }}$. Please note that Einstein's summation convention leads to a summation over identical indices in an expression, for example, $u_{k, k}=u_{x, x}+u_{y, y}+u_{z, z}$. Here, $\delta_{m n}$ denotes the Kronecker delta.

The material behavior $\alpha(\vartheta)$ is calibrated with experimental data of free swelling and the superposition with mechanically induced strains allows the simulation of arbitrary hydrogel geometries in free or clamped configurations. Studies have shown that the displacement of the hydrogel pore structure is independent of the Young's modulus in the elasticity tensor $E_{k l m n}$, but depends on Poisson's ratio $\nu$. Simulations using the described model were performed by using the Finite Element tool Abaqus. They form the basis of the following fluidic investigations.

Geometries. In our previous work, different pore shapes were investigated to assess their individual performance for a size-exclusion system. The four different pore shapes depicted in Figure 4 are used in the current work.

1. Case I: The round pore serves as an analytical reference since due to radial symmetry, both the swelling behavior and the flow profiles can be calculated in an easy way.

2. Case II: The rectangular pore is the typical noncircular channel geometry. For this case, the hydraulic diameter can be used without additional correction factors to gain the volume flux. In the current work, Case IIa is a quadratic pore and IIb a rectangular pore with the width being twice the height.

3. Case III: The cross-shaped pore is composed of two orthogonal rectangles. It offers the best opening/closing abilities as concluded in Ehrenhofer et al. (2016b).

4. Case IV: The triangle pore offers good opening/ closing performance combined with a good bypass of particles and fluid in the blocked state.

The main target of the current work is to model the flow through a permeation control membrane with
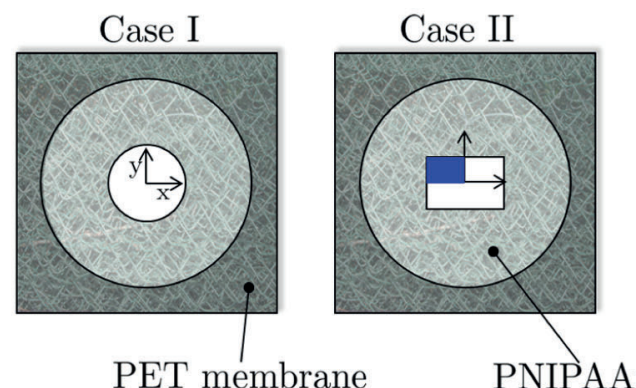

Case II

PNIPAAm

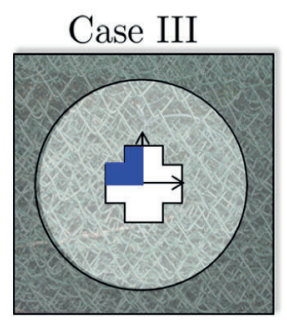

Simulation region

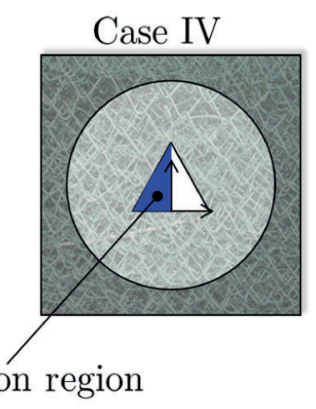

Figure 4. Setup for pores of different geometries in a polyethylene terephthalate foil. The simulation region is the inside of the pore where the fluid flux occurs. Symmetry is used where appropriate to reduce computational costs. 


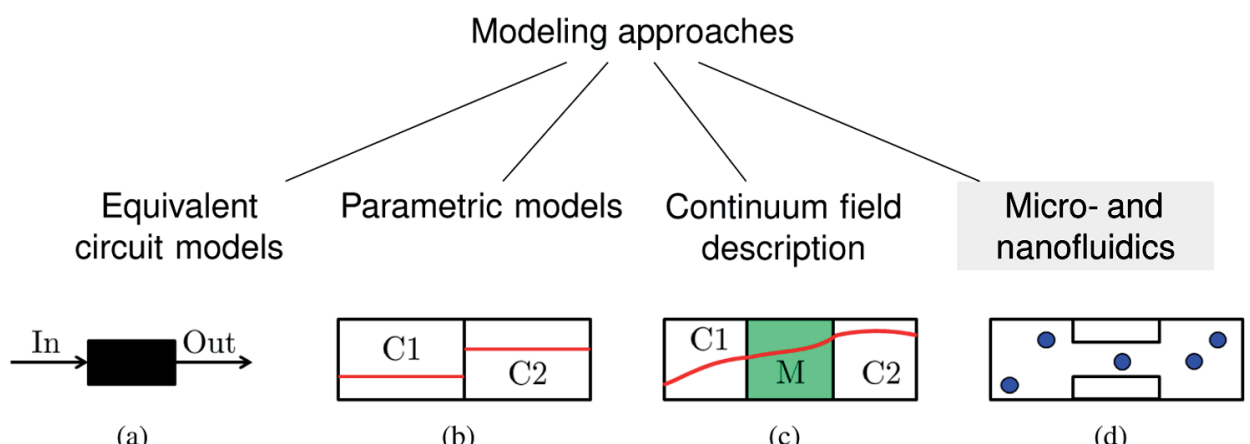

Figure 5. Ion permeation through membranes can be modeled on different accuracy levels regarding the resolution of physical effects. The current work uses a description of the flow on the microfluidic level (d).

different pore geometries. It serves a better understanding of the ongoing processes regarding pressure loss and flow profiles. With this knowledge, a fluid structure-interaction simulation can be performed. Therefore, a microfluidic model for the fluid flow through the membrane is depicted, see section 'Methods for modeling of membrane fluid permeation'. The implementation of the model and results of the simulations are shown in section 'Results of the numerical simulations'. The conclusion is drawn in section 'Conclusion and outlook'.

\section{Methods for modeling of membrane fluid permeation}

Fluid permeation through membrane pores can be modeled on different levels. These are: (a) equivalent circuit models, (b) parametric models, (c) continuum models, (d) microfludic/nanofluidic models, see Figure 5. Models for biological membranes mostly focus on the gating behavior of channels for ions, while models for polymeric membranes focus on the pressuredriven flow and separation. While (a) equivalent circuit models or network models are based on black box descriptions characterized by the transfer function, the class of (b) parametric models takes discrete compartments with physically derived balance equations into account. (c) Continuum models can give an insight into the spatial distribution of field variables like ion concentrations, the membrane itself is modeled as a region of altered material properties. (d) The microfluidic and nanofluidics approaches are based on the description of flow inside the membrane pores. If the dominating effects can be described through continuum theory, the models are microfluidic. For smaller scale effects, the nanofluidic modeling with stochastic dynamics or molecular dynamics is used.

In the present work, we investigate the particle flow through a smart membrane pore on the microfluidic level. The flow of solvent molecules is described on the basis of continuum mechanics using the Navier-Stokes equations in their simplified form, see section 'Governing equations'. This is allowed because, in the current work, the limits of continuum theory are not exceeded for the pores (Daiguji, 2010). The channel walls are considered rigid, that is, the pressure exercised by the fluid does not lead to a deformation of the pore structure. Additional studies using Fluid-Structure Interaction (FSI) methods will be necessary when higher pressure differences over the membrane are inflicted, which lead to a bending of the composite membrane.

A closer look into the influencing effects with the help of the theory of laminar flows is taken in section 'Influencing fluidic effects'. The analytical derivations on the basis of Bernoulli's equation lead to the result, that the flow profiles resulting from a pressure gradient over the membrane can be calculated. The respective model is explained in section 'Flow profiles'. Results of the numerical simulations for open and blocked pores are shown in section 'Results of the numerical simulations'.

\section{Governing equations}

The fluid flow through every single pore can be described as a Hagen-Poiseuille flow in a non-circular channel. It can be characterized as an incompressible creeping (and hence laminar) flow (Squires and Quake, 2005) because of the miniature characteristic dimensions (e.g. the pore diameter $d_{\text {Pore) }}$ of the pore system and subsequent low Reynolds number

$$
\operatorname{Re}=\frac{d_{\text {Pore }} \bar{v}}{\eta} \ll 1
$$

Here, $\bar{v}$ denotes the mean fluid velocity through the pore and $\eta$ the dynamic viscosity of the fluid.

The governing equations of a general monophasic flow (Kirby, 2010) are the continuity equation derived from the mass balance 


$$
\frac{\partial \rho}{\partial t}+\left(\rho v_{i}\right)_{, i}=0
$$

with the density $\rho$ and the velocities $v_{i}$ with $i \in[x, y, z]$ as well as the momentum equations

$$
\rho \frac{D v_{i}}{D t}=-p_{, i}+\tau_{i j, j}+\rho f_{i}
$$

with the pressure $p$, the stress tensor $\tau_{i j}$, body forces $f_{i}$ and the material time derivative $D / D t$. Newton's stress hypothesis yields

$$
\tau_{i j}=\eta\left(v_{i, j}+v_{j, i}-\frac{2}{3} \delta_{i j} v_{k, k}\right)+\eta^{\prime} \delta_{i j} v_{k, k}
$$

with the volume viscosity $\eta^{\prime}$ denoting the fluid's resistance to compression. Together with Stokes' hypothesis $\eta^{\prime}=0$, equations (5) and (6) are called NavierStokes equations. They are valid for Newtonian fluids and form a parabolic-elliptic system of partial differential equations. Here, $\partial_{t}$ denotes the local time derivative $\partial / \partial t$

$$
\begin{aligned}
& \partial_{t} \rho+\left(\rho v_{i}\right)_{, i}=0 \\
& \partial_{t} v_{i}+v_{j} v_{i, j}=-\frac{1}{\rho} p_{, i}+f_{i}+\frac{\eta}{\rho}\left(v_{i, j}+v_{j, i}-\frac{2}{3} \delta_{i j} v_{k, k}\right)_{, j}
\end{aligned}
$$

\section{Influencing fluidic effects}

For the sake of estimating the influence on the pressure loss in a switching pore of any geometry, we look at a circular pipe using the theory of laminar flows. From the energy conservation on a streamline with arc coordinate $s$ between two points (1) and (2) in integral form, the Bernoulli equation

$$
\int_{s_{1}}^{s_{2}} \frac{\partial v_{s}}{\partial t} d s+\left.\frac{v_{s}^{2}}{2}\right|_{1} ^{2}+\int_{p_{1}}^{p_{2}} \frac{1}{\rho} d p+\left.U\right|_{1} ^{2}+\frac{\Delta p_{\text {loss }}}{\rho}=0
$$

with superimposed pressure loss (or head loss) $\Delta p_{\text {loss }}$ due to fluidic resistances can be derived. $v_{s}$ denotes the fluid velocity in the direction of the streamline. The difference of the potential energy $U$ can be neglected in horizontal alignment. Also stationary flow is considered. Points on the streamline with the arc length coordinate $s$ are the inlet $\mathbf{I}$, the pore $\mathbf{P}$ and the outlet $(\mathbf{O}$. The overall pressure loss of the pore-membrane is dependent on three major mechanisms (see Figure 6)

$$
\Delta p_{\text {loss }}=\Delta p_{\text {loss }, \text { Inlet }}+\Delta p_{\text {loss, Pore }}+\Delta p_{\text {loss }, \text { Outlet }}
$$

The velocities in the pore and outlet region can be calculated with the continuity equation (5).

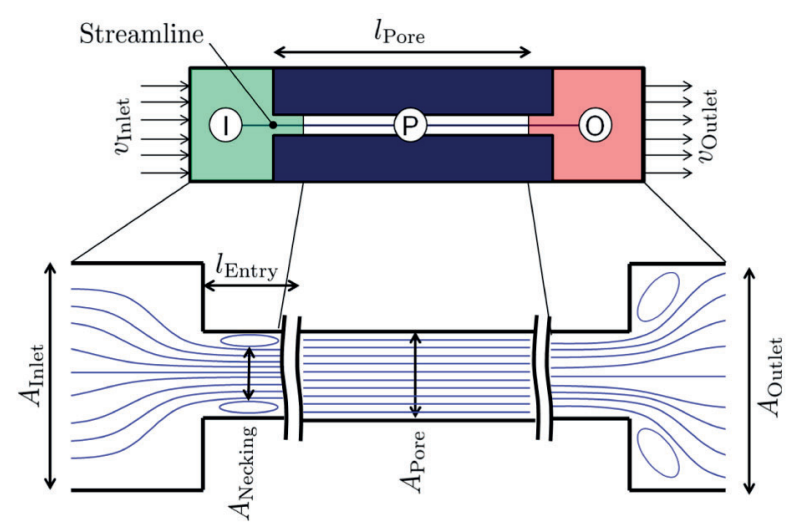

Figure 6. Streamlines for a general flow through a pore. It can be subdivided into the inlet, a region of undisturbed pipe flow and the outlet. Points inside the respective regions are (I), (P) and $(\mathrm{O})$.

Inlet. At the inlet of a pore, a pressure loss due to the necking of the stream occurs. The area $A_{\text {Necking }}$ to which the stream is restricted can be calculated using the contraction number $\alpha_{\mathrm{n}}=A_{\text {Necking }} / A_{\text {Pore }}$. It is very similar for circular apertures $\alpha_{\mathrm{n} \text {,circular }} \simeq 0.61$ and parallel plates $\alpha_{\mathrm{n} \text {,parallel }} \simeq 0.58$, and can therefore be used to estimate the inlet pressure loss for an arbitrary pore geometry

$$
\Delta p_{\text {loss }, \text { Inlet }}=\rho \frac{v_{\text {Pore }}^{2}}{2}\left(\frac{1-\alpha_{\mathrm{n}}}{\alpha_{\mathrm{n}}}\right)^{2}
$$

The inlet length - until the laminar flow is completely developed $(d u / d z=0)$ - depends on the pore Reynolds number $R e_{\text {Pore }}=v_{\text {Pore }} d_{\text {Pore }} / \nu$ with $\nu=\eta / \rho$ being the kinematic viscosity of the fluid. This leads to an entry length of

$$
l_{\mathrm{E}, \text { laminar }}=\frac{R e_{\text {Pore }}}{32} d_{\text {Pore }}
$$

Due to the very low Reynolds number (equation (4)), the entry length $l_{\mathrm{E}}$ is much smaller than the pore length $l_{\text {Pore }}$ which is the overall thickness of the composite membrane

$$
\frac{l_{\text {E,laminar }}}{l_{\text {Pore }}}=\frac{R e_{\text {Pore }} d_{\text {Pore }}}{32 l_{\text {Pore }}} \ll 1
$$

Therefore, in the pipe friction part the overall pore length as a region of laminar, developed flow can be used.

Pipe friction. In the part of the pore where laminar and developed flow is assumed, the pressure loss can be calculated from the pipe flow coefficient (Darcy friction factor)

$$
\lambda_{\text {laminar,developed }}=\frac{64}{R e_{\text {Pore }}}
$$


The overall pressure loss in the pore takes this coefficient and the kinetic energy of the flow into account. The Darcy-Weisbach equation

$$
\Delta p_{\text {loss, Pore }}=\lambda \frac{l_{\mathrm{P}}}{d_{\mathrm{P}}} \frac{\rho}{2} v_{\mathrm{P}}^{2}
$$

with the respective coefficient $\lambda=\lambda_{\text {laminar, developed }}$ can be used to assess the influence of this part in the overall pressure loss in comparison to inlet and outlet.

Outlet. The outlet can be characterized as a diffuser with an opening of $90^{\circ}$. Then, the pressure loss can be described with the Borda-Carnot equation

$$
\Delta p_{\text {loss }, \text { Outlet }}=\frac{\rho}{2}\left(v_{\text {pore }}-v_{\text {out }}\right)^{2}
$$

Typical velocities for microfluidic devices range from $1 \times 10^{-6} \mathrm{~m} / \mathrm{s}$ to $1 \times 10^{-2} \mathrm{~m} / \mathrm{s}$. Pressures up to $140 \mathrm{~Pa}$ can be found (Squires and Quake, 2005). In the present work, a smart membrane system with a single pore (highest fluid velocity) and an opening range of $50 \mu \mathrm{m}$ to $200 \mu \mathrm{m}$ is considered. The comparison of pressure losses at the inlet $p_{\text {loss,Inlet, outlet }} p_{\text {loss, Outlet }}$ and due to pipe friction $p_{\text {loss,Pore }}$ shows that the pressure loss inside the pore is always dominating for microfluidic flows in our device.

\section{Single pore versus membrane system}

The number of pores in the membrane $N_{\text {Pores }}$ plays a role in the fluidic behavior since, due to the continuity equation (5), more pores lead to a decreased velocity in every pore. Expressed in the analogy of fluidic resistances (Kirby, 2010; Paschew et al., 2016), many pores in a membrane can be seen as a parallel setup. When a pore is blocked, its individual resistance increases. The overall flow through a massive parallelized membrane system can best be addressed with network models.

The current work deals with the flow through a single pore in free and blocked form, see Figure 7. The blocking of a pore is discrete while the opening and closing is continuous as described in Figure 3.

\section{Flow profiles}

The previous section has shown that inlet and outlet pressure losses at the membrane are irrelevant for microfluidic flows. Calculating the flow profiles through the pore in any open or closed state must thus concentrate on the flow through the pore itself.

The kinematic assumptions of unidirectional flows in non-circular channels lead to simplified equations for the velocity profile in the pore, that is, the Poisson's equation of the Poiseuille flow

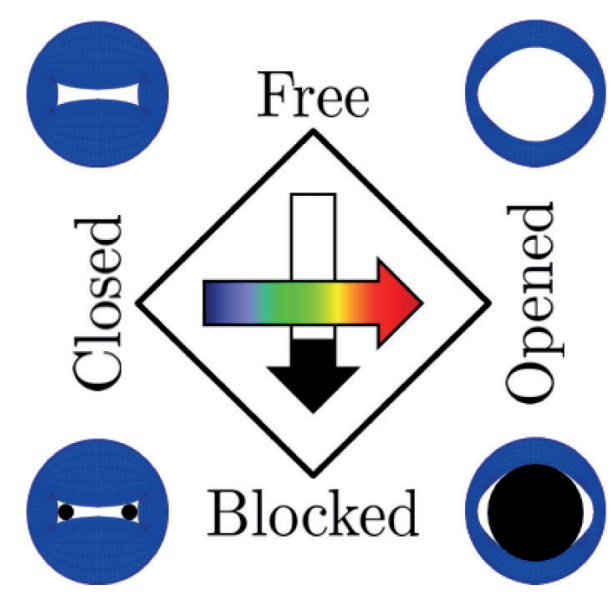

Figure 7. The flow through a single pore is influenced by its continuous opening state, which depends on the temperature and its discrete state of being blocked by a particle. For the rectangle shaped pore depicted here, two regions of particles can be identified in which the stream can be blocked by particles.

$$
\nabla^{2} v(x, y)=-\frac{K}{\eta}
$$

where $K=d p / d z$ denotes the constant pressure gradient throughout the channel. The Poisson's equation (17) can be solved by giving appropriate boundary conditions, which is the no slip condition $\left.v(x, y)\right|_{\text {wall }}=0$ at wetting walls or ambient pressure at free surfaces. The shape of the wall can be extracted from the Finite Element solution of the swelling problem of the switchable pore (Ehrenhofer et al., 2016b). This can be performed for an arbitrary pore geometry and openingstate. The volume flux is then calculated by

$$
\dot{V}=\int_{A} v(x, y) d A
$$

The mean velocity $\bar{v}=\dot{V} / A$ together with the diameter of blocking particles is used to characterize the overall performance of the membrane with respect to water flow and filtration.

\section{Bypass of particles and fluid}

A crucial property characterizing the functionality of the smart membrane in a particle size sequencing system is the bypass. When a particle flow is led through a membrane consisting only of round pores, this would lead to a breakdown of the overall flow. Also, after the clogging of the membrane, no particles would be allowed to pass through the membrane. These particles, called bypassers henceforth, and the according bypasser-diameter $d_{\text {Bypasser }}$ limit the detection precision that the smart membrane system can achieve: All particles of diameters $d_{\text {Particle, }}$, between the blocking particles 


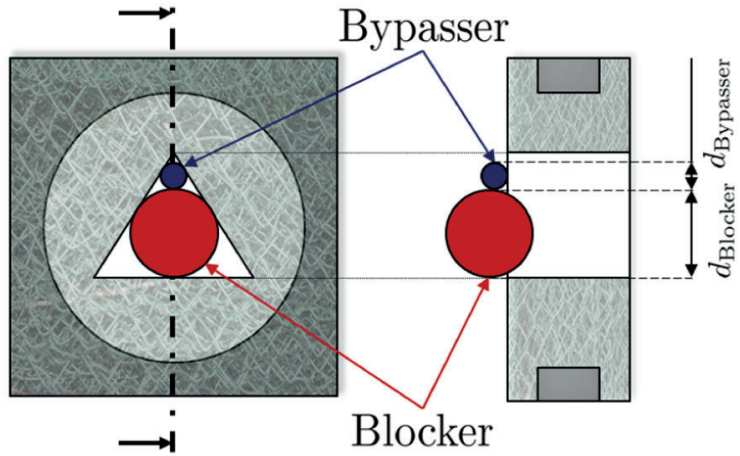

Figure 8. Blocking and bypassing particles with their respective diameters for the triangle shaped pore in reference state. Bypassers are the next bigger particles that can pass through the bypass-area created by the blocker. For different shapes and opening states there could be more than one bypasser particle.

(called blockers with $d_{\text {Blocker }}$ henceforth) and the bypasser-diameter, are blocked as well. This does not happen if there are more unblocked pores than blocking particles, which may be hard to achieve in native biological substances like blood. The concept of blockers and bypassers is depicted in Figure 8. There, it can also be seen that depending on the chosen shape, a different number of particles can pass by.

\section{Results of the numerical simulations}

To get an insight into the flow through pores at any opening state in the free or blocked condition, numerical simulations were performed. The dimensions of the different pore shapes (see Figure 4) were chosen with the same hydraulic diameter $d_{\mathrm{h}}=4 A / C=$ $85.8447 \mu \mathrm{m}$ in reference configuration. This geometry value for non-circular channel cross sections is composed of the area $A$ and the circumference $C$ and denotes the diameter of a circle with equivalent flow. The shape change was simulated according to the Temperature Expansion Model described in our previous works (Ehrenhofer et al., 2016a,b). The flow through the membrane could be either (i) under constant pressure gradient across the pore with the volume flux as the measurement variable, or (ii) under constant flow rate with measured pressure drop. Here, a constant pressure difference of $\Delta p=12 \mathrm{~Pa}$ over the pore length of $l_{\text {Pore }}=100 \mu \mathrm{m}$ is assumed, and the volume flux over the membrane is calculated with the Poisson's equation (17). The temperature-induced change of the dynamic viscosity of water $\eta \approx \eta_{0}=1 \times 10^{-3}$ Pas was not taken into account, since its impact on the overall flux is very small in comparison to the impact of the pore size change. The partial differential equation was solved using the Matlab PDEtool solver which uses the Finite Element Method. To reduce numerical costs, only a quarter of the rectangle, square and cross pores and a half pore for the triangle were simulated. Boundary conditions were (a) Neumann conditions at the symmetry planes and (b) Dirichlet no slip conditions at the borders of the fluidic area, which is the hydrogel pore structure. Observed fluxes $\dot{V}$ were up to $6000 \mathrm{nl} \mathrm{s}^{-1}$ $\left(=6 \times 10^{-6} 1 \mathrm{~s}^{-1}\right)$ with maximal flow velocities $v_{\max }$ of $0.3 \mathrm{~m} \mathrm{~s}^{-1}$. In microfluidics, Zhang et al. (2015) use higher pressures of up to $50 \mathrm{kPa}$ while Paschew et al. (2016) use lower pressures up to 150 mbar. For defining a working point of the envisioned microfluidic size sequencing device, a better understanding of the composite membrane's mechanics is needed, which will be subject to our future work. In the following, the results for each pore type are shown separately and then discussed together.

\section{Case I: Round pore}

The round pore is the simplest geometrical shape, both in manufacturing and in simulation treatment. In cylindrical coordinates $[r, \varphi, z]$, assuming circular symmetry $v \neq v(\varphi)$, equation (17) is simplified to

$$
\frac{1}{r} \frac{d}{d r}\left[r \frac{d v(r, \varphi)}{d r}\right]=-\frac{K}{\eta}
$$

Together with the no-slip boundary condition $v(r=R)=0$ at the outer border $r=R$, which is also the inner border of the perforated disc (hydrogel region), the quadratic flow profile

$$
v(r, \varphi)=\frac{K}{4 \eta}\left(R^{2}-r^{2}\right)
$$

follows. This, together with equation (18), leads to a total volume flux of

$$
\dot{V}=\frac{\pi R^{4} K}{8 \eta}
$$

This equation is known as the Hagen-Poiseuille equation. For the analytical solution of the opening and closing mechanics of the pore, equations (1) to (3) are combined, transformed to polar coordinates and simplified to the one-dimensional form through rotational symmetry. As described in Ehrenhofer et al. (2016b), the radial displacement $u_{r}$ of the disc can then be calculated by

$$
\frac{d}{d r}\left[\frac{1}{r} \frac{d\left(r u_{r}\right)}{d r}\right]=(1+\nu) \alpha(\vartheta) \frac{d(\Delta \vartheta)}{d r}
$$

with $\Delta \vartheta=\vartheta-\vartheta_{0}$ being the difference of the temperature $\vartheta$ in $\mathrm{K}$ to the reference (Ehrenhofer et al., 2016b). Please note that the temperature $\vartheta$ is given in ${ }^{\circ} \mathrm{C}$. The thermal expansion coefficient $\alpha(\vartheta)$ is calibrated by the free swelling behavior of the hydrogel material in the temperature range. The boundary conditions are (a) fixed at the contact to the outer polyethylene 
terephthalate (PET) foil $\left.u_{r}\right|_{\text {outer }}=0$ and (b) a free boundary at the inside $\left.\sigma_{r}\right|_{\text {inner }}=\sigma_{r}(r=R)=0$, where the hydrogel is in contact with the fluid.

The analytical derivations predict a high flow through round pores. At the same time, the shape change under temperature stimulus is much smaller than for the other shapes. This leads to a change in flux versus temperature for the round pore that is overall much smaller than for the other geometries, see below.

\section{Case II: Rectangular pore}

The rectangular pore shape has a special case, which is the square. Simulations were performed both for a rectangular shape where the width is twice the height, and the square. The rectangle pore offers larger displacements than the square pore, therefore the maximum flux $\dot{V}$ is higher for the rectangle pore as well. When pores of rectangular geometry close from the reference state, the flanks get in contact and the area where flow can pass through diminishes, see Figure 9 (Rectangle free, $\vartheta=23^{\circ} \mathrm{C}$ ). Both shapes converge to a circle when opening (Rectangle and Square free, $\vartheta=38^{\circ} \mathrm{C}$ ). This leads to a reduced bypass flux when they are blocked by particles. On closing, the middle section of the rectangle shows larger displacement than the sides which leaves space for two particles as blockers that are not at the center, but at the sides (Rectangle blocked, $\left.\vartheta=23^{\circ} \mathrm{C}\right)$.

\section{Case III: Cross pore}

The cross shaped pore consists of two orthogonal rectangles and thus shows a similar behavior to a simple rectangle, that is, the cross arms like the sides of the rectangle allow a bypass of flux and particles. When closing, four particles can be blocked at the arms, see Figure 10 (Cross blocked, $\vartheta=23^{\circ} \mathrm{C}$ ). On opening, the structure nears the circular shape as well. In contrast to the simple rectangle, the edges between the former cross flanks are the contact points to a blocking particle so that a higher bypass flux can occur (Cross blocked, $\left.\vartheta=38^{\circ} \mathrm{C}\right)$.

\section{Case IV: Triangle pore}

The triangular pore shape was chosen since it offers the property that when it is blocked, the remaining bypass areas are themselves triangular shaped. The bypass diameter for the next smaller level is about 0.330 times the diameter of the actual blocker and three particles can pass by this way. This property is fractal in nature; for each bypasser, another three particles with a third of the size can pass. In addition, the pore preserves its triangular shape for all closing states, that is, the triangle shape can still be found in the deformed shape. It thus offers the respective property throughout the closing process, see Figure 10 (Triangle free, $\vartheta=23^{\circ} \mathrm{C}$ ).

\section{Comparison of the different pore shapes}

Free state. As expected, in free state, the different pore shapes enable different fluxes when opening and closing over the temperature range. The differential equation describing the fluid flux through the free pores yields that a correction of the hydraulic diameter is needed to gain the adequate flux in reference state $\dot{V}=f\left(d_{\mathrm{h}}, k\right)$. Since no such corrections have been performed, $\dot{V}_{\text {free }}\left(\vartheta=32^{\circ} \mathrm{C}\right)$ is not equal for all geometries, see Figure 11(a). Nevertheless, these differences in reference state are small compared to the change over temperature, thus a comparison and assessment of the shapes can be drawn from the simulations.

Due to the much larger change of passage diameter over temperature and thus also bigger change of the flow area, the cross pore offers the best permeation control abilities. When regarding the free flux over the passage diameter $\dot{V}_{\text {free }}\left(d_{\text {Passage }}(\vartheta)\right)$ (see Figure $11(\mathrm{~b})$ ), the cross pore performs best as well. In course of the opening, that is, for $\vartheta>\vartheta_{\text {ref }}=32^{\circ} \mathrm{C}$ all shapes become nearly circular since this is the form of the outer restriction of the swelling hydrogel.

Blocked state. For a circular pore no bypass flux is observed, that is, $\dot{V}_{\text {Bypass,Circular }}=0$. For other pore geometries, the bypass flux diminishes when the pores approach a nearly circular shape in course of opening. The state when this happens can be identified in Figure 12 (a) for temperatures $\vartheta>33^{\circ} \mathrm{C}$. There, the bypass significantly drops. As a good bypass for particles and fluid is needed, the preferable temperature range for the device is limited. In Figure 12(b), the flux over the size of the blocking particle - in this case the passage diameter $d_{\text {Blocker }}=d_{\text {Passage }}$-is depicted. The jumps in flow occur when the position of the passage diameter changes from the center to the sides, see Figure 9 (Rectangle blocked $23^{\circ} \mathrm{C}$ and $32^{\circ} \mathrm{C}$ ). Then, for some geometries, a larger number of equally sized particles can act as blockers, that is, $N_{\text {Blocker }}>1$. For the rectangle this is $N_{\text {Blocker,max }}=2$ and for the cross $N_{\text {Blocker,max }}=4$. For such a blocked pore with $N_{\text {Blocker }}$ $<N_{\text {Blocker,max }}$, the flux can be calculated through the share of the blocked area for the flux

$$
\begin{aligned}
\dot{V}_{\text {Partly blocked }}= & \frac{N_{\text {Blocker }}}{N_{\text {Blocker,max }}} \dot{V}_{\text {Bypass }} \\
& +\frac{N_{\text {Blocker,max }}-N_{\text {Blocker }}}{N_{\text {Blocker,max }}} \dot{V}_{\text {free }}
\end{aligned}
$$

In the present work, we consider that a pore is blocked by the maximum number of particles. 


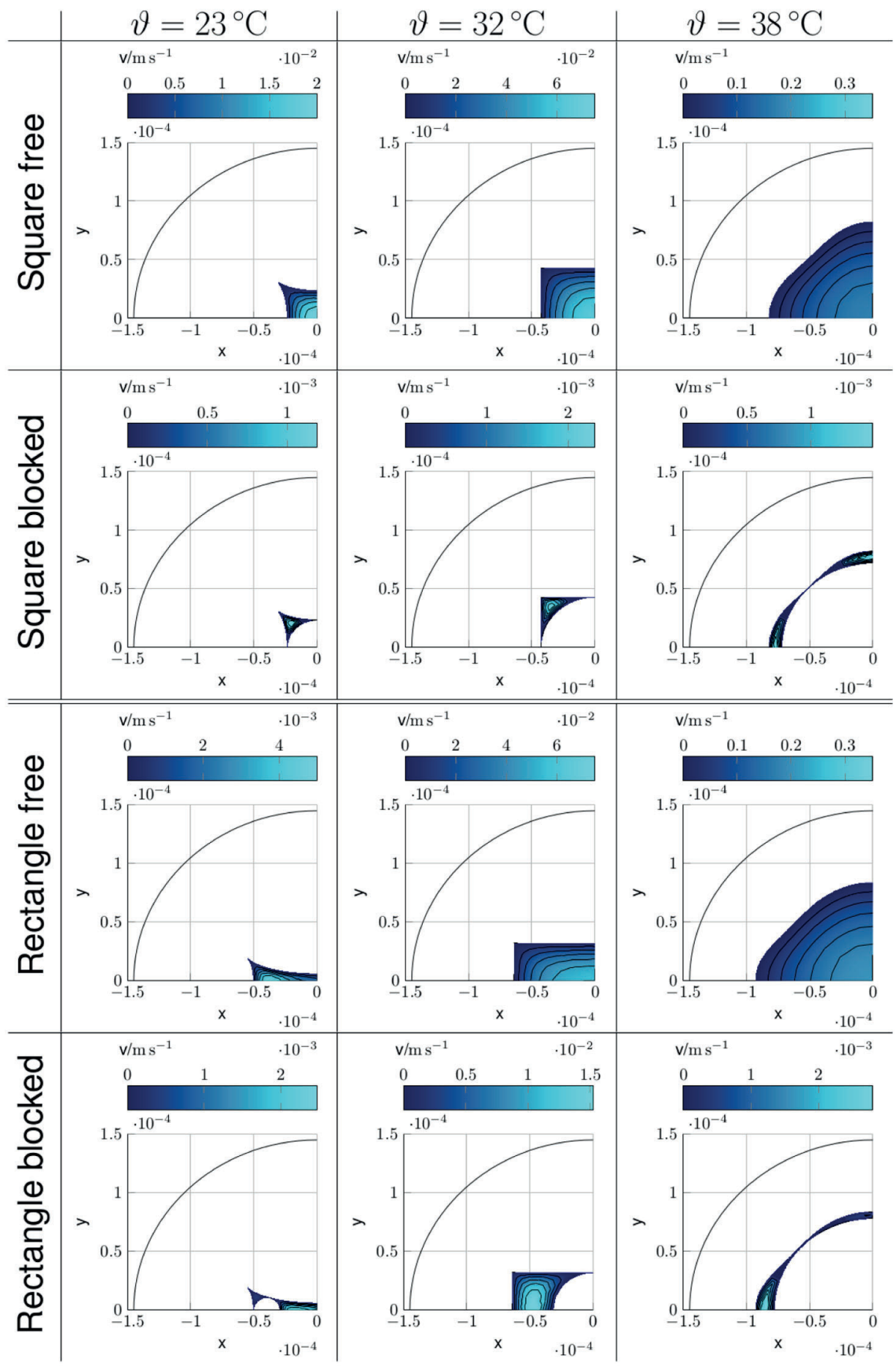

Figure 9. Geometry shapes and flow at different temperatures in the free and blocked state for the square and rectangle pore. The outer circle denotes the position of the fixing between PET membrane and hydrogel. The $x$ - and $y$-axes are symmetry axes; only a quarter pore is simulated. Please note the different color bars for the different setups.

The distinguishing parameter for the final function of the size sequencing system is the flow difference between the free and blocked pores. When particles with a diameter larger than the passage diameter are present inside the stream, the equivalent number of pores is blocked by those particles. Then, particles smaller than the bypass diameter $d_{\text {Bypass }}$ are not blocked. From the flux difference at any opening/closing state, which amounts when the membrane pores are blocked, the number of particles larger than the actual 


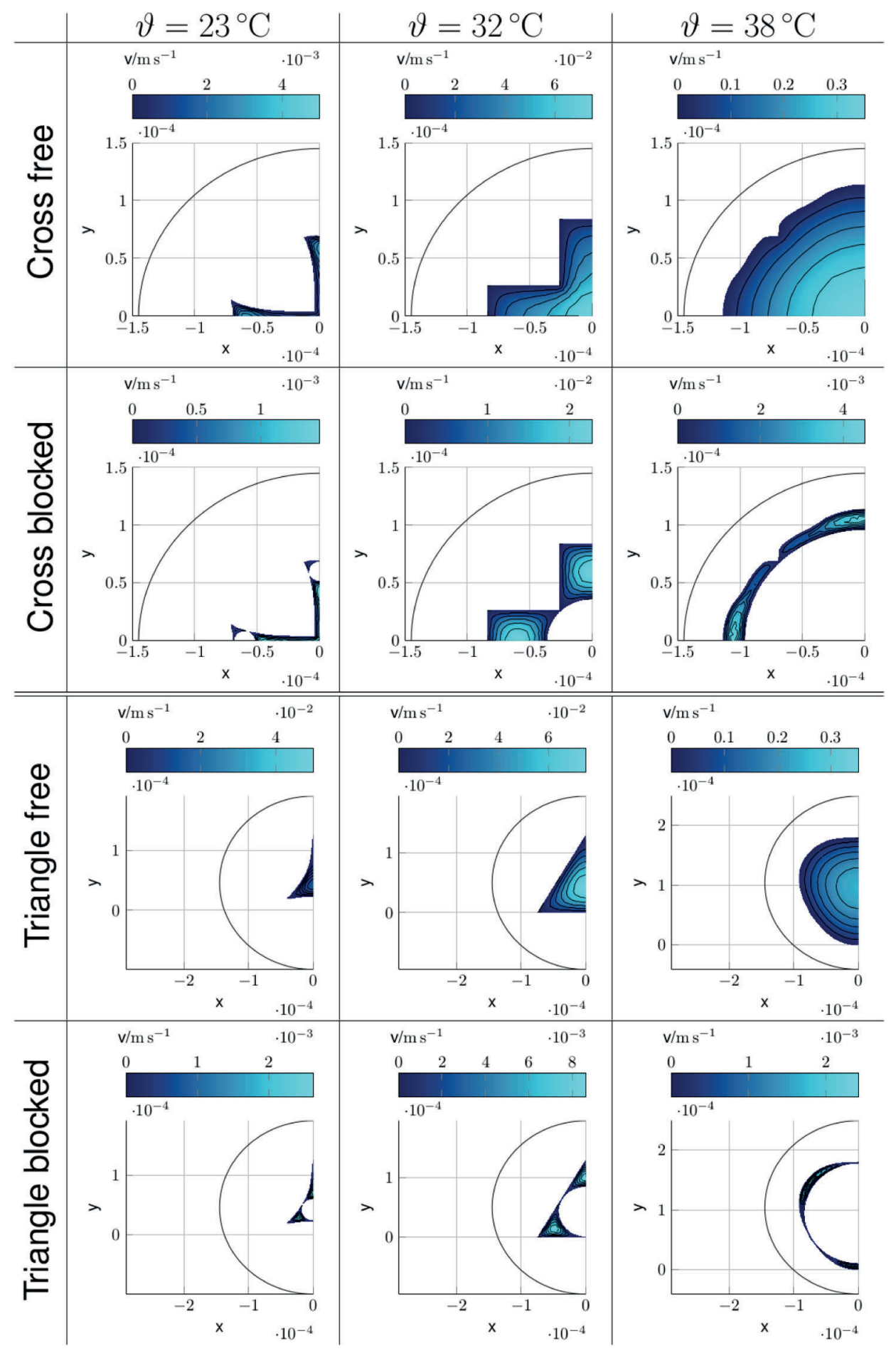

Figure 10. Geometry shapes and flow at different temperatures in the free and blocked state for the cross and triangle pore. The outer circle denotes the position of the fixing between PET membrane and hydrogel. For the triangle pore, the half setup was simulated. Please note the different color bars for the different setups.

passage diameter can be calculated. This only holds true for rigid particles and if all other particles can bypass the blocked ones.

From the simulations performed in the course of this study, it is observed that the flux substantially diminishes when the pore is blocked by a particle of the adequate size. The normalized flux drop, that is, the flux difference between a free and completely blocked pore related to the free flux $\left|\dot{V}_{\text {free }}-\dot{V}_{\text {Blocked }}\right| / \dot{V}_{\text {freee }}$, is depicted in Figure 13 for all investigated pore 


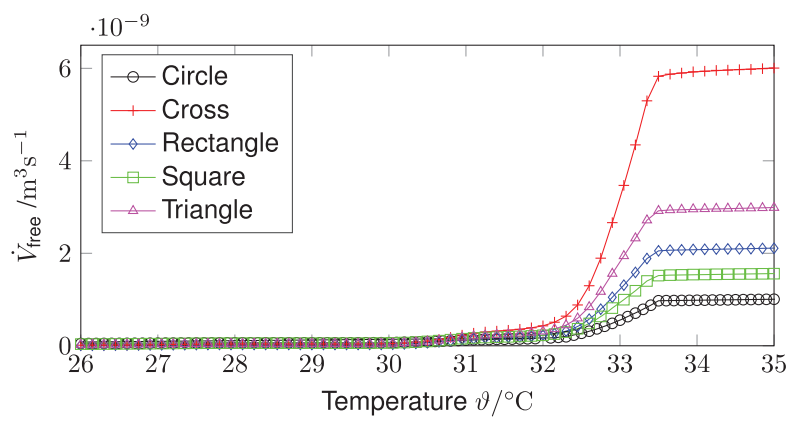

(a) Free flux versus the temperature. The simulations were performed in the temperature range from $23{ }^{\circ} \mathrm{C}$ to $38^{\circ} \mathrm{C}$. The results are depicted in a smaller range since the change is insignificant elsewhere.

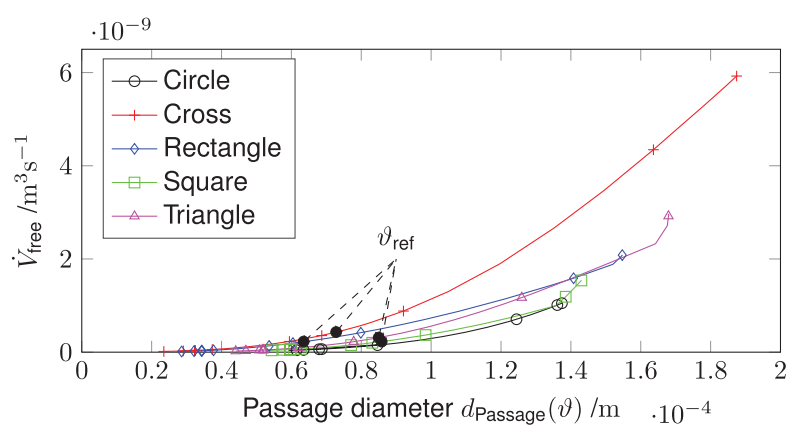

(b) Free flux versus the passage diameter, i.e. the largest particles that can pass through the pore. Due to the different opening/closing abilities of the geometries, the ranges in $d_{\text {Passage }}$ differ. Please note that at the reference temperature, different passage diameters can be found.

Figure II. Free volume flux $\dot{V}_{\text {free }}$ through the pores at various opening states versus the temperature II (a) and the respective passage diameter II (b). For temperatures $\vartheta>\vartheta_{\text {ref }}=32^{\circ} \mathrm{C}$, the pores open and larger particles can pass through.

geometries. When the flux through a membrane with switchable pores at constant pressure gradient is measured, the number of blocked pores with $N_{\text {Blocker,max }}$ can be calculated as follows.

1. A constant pressure difference $\Delta p_{\text {Measurement }}$ is applied over the membrane and the flux $\dot{V}_{\text {Out }}$ is measured.

2. The fluid including the particles to measure is temperated to $\vartheta_{\text {Measurement }}$.

3. The passage diameter $d_{\text {Passage }}(\vartheta)$ can be read from the simulations in Ehrenhofer et al. (2016b) or through the measured flux in Figure 11(b). All particles with larger sizes will be blocked.

4. For each blocked pore, a flux drop according to Figure 13 will occur. From $\dot{V}_{\text {Out }}$, the number of blocked pores can thus be calculated.

5. Respecting $N_{\text {Blockers, }}$, this is the number of particles with the size larger or equal to $d_{\text {Passage }}(\vartheta)$.

6. Varying $\vartheta_{\text {Measurement }}$ gives the size distribution of particles in the flow.

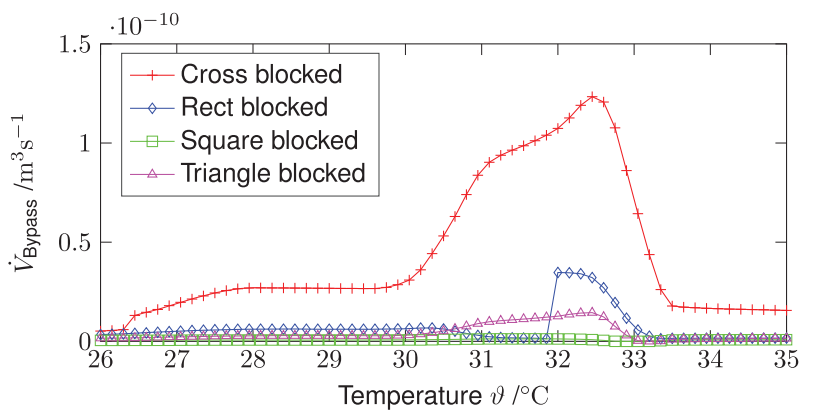

(a) Bypass flux versus temperature. Simulations were performed in the temperature range from $23{ }^{\circ} \mathrm{C}$ to $38^{\circ} \mathrm{C}$. The results are depicted in a smaller range since the change is insignificant elsewhere.

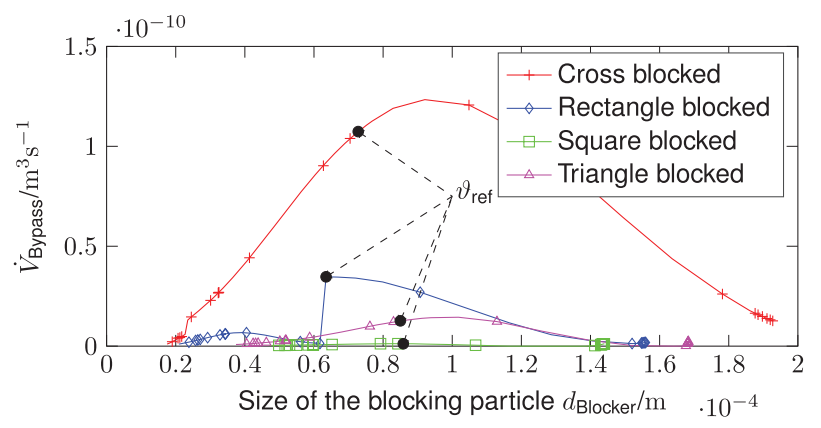

(b) Bypass flux versus blocker diameter. For $\vartheta<\vartheta$ ref and subsequent smaller particle diameters, different fluxes according to the number of blocking particles can occur, see equation (23). Please note that $d_{\text {Blocker }}$

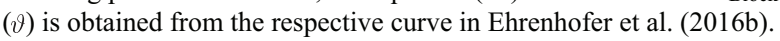

Figure 12. Bypass flow $\dot{V}_{\text {Bypass }}$ through the pore when it is blocked at temperature $\vartheta$ (a) or by a particle the size of $d_{\text {passage }}$ (b). The circular pore is left out because no bypass can occur in the blocked state.

When more than one pore is used to form a membrane system, a model of fluidic resistances as described in section 'Single pore versus membrane system' can be used. The derived characteristics for each pore as presented in the current work can be used to define these resistances.

\section{Conclusion and outlook}

The hydrogel composite membrane described in the present work can be used to measure size distributions in a particle fluid flow. For the functioning of such a system, the bypass of particles and fluid in a blocked pore is of primary importance. The simulations of the shape change for different pore geometries performed in our previous work was extended to allow the assessment of flow, blocking and bypass. Studies based on the concept of streamlines and on the Bernoulli equation in a pressure-driven microfluidic flow were performed. The analytical derivations have shown that the problem can be reduced to a unidirectional flow inside the pore. The flow at the inlet side in free, partially 


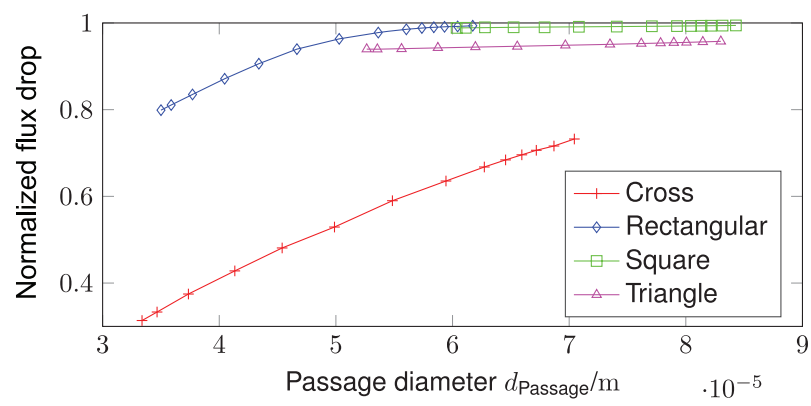

Figure 13. Normalized flux drop $\left|\dot{V}_{\text {free }}-\dot{V}_{\text {Blocked }}\right| / \dot{V}_{\text {free }}$ versus the passage diameter $d_{\text {passage. }}$. The flux drop is the change of flux between a free and completely blocked pore normalized by the free flux. Please note that only values in the closing direction, that is, $\vartheta \leqslant \vartheta_{\text {ref }}$ are depicted, since this is the desired shape changing range.

blocked or completely blocked state can then be predicted using the Poisson's equation of Poiseuille flow. Three-dimensional computational fluid dynamics simulations at a blocked inlet can show if this reduction of the problem holds.

Simulation results from the pore shape change show the effect that an opening pore becomes nearly circular when the outer fixing of the hydrogel to the support membrane is of circular shape. When a nearly circular pore is blocked, the bypass of fluid and particles is much lower than, for example, in cross shaped pores. The opening of the pores until the shape becomes nearly circular is therefore not preferable. Hence, the stimulus range for the switching must be limited. Alternatively, pore shapes that do not change to a circle can be created and optimized. The described Temperature Expansion Model for hydrogel swelling allows investigation and design of such shapepreserving pores.

When constant pressure is applied and the pore is switched inside the preferable stimulus range, that is, when bypass occurs, the simulations predict the diminishing flow. The change of the output value in a microfluidic particle size sequencing system can directly be used to compute the number of blocking particles of the size-range preselected through the temperature.

Additional influencing effects like the fluid structure interaction of soft particles as well as the strains due to bending of the membrane will be incorporated into our model in order to reach the goal: The design of a microfluidic membrane device that can measure the cell size distribution in native biological fluids like blood. The simulations of the fluxes through the smart membrane system that were performed in the current work give a closer insight into the function and limits of a particle size sequencing system based on those membranes.

\section{Declaration of conflicting interest}

The author(s) declared no potential conflicts of interest with respect to the research, authorship, and/or publication of this article.

\section{Funding}

The author(s) disclosed receipt of the following financial support for the research, authorship, and/or publication of this article: The present work is supported by the Excellence Initiative by the German Federal and State Governments in the framework of the Institutional Strategy 'The Synergetic University' within the project 'Numerische und experimentelle Untersuchungen zur schaltbaren Permeabilität von Doppellipidschichten als intelligente Strukturen' at Technische Universität Dresden. It is also partly supported by the Center for Advancing Electronics Dresden (cfaed) at Technische Universität Dresden and by the Research Training Group DFG-GRK 1865 'Hydrogel-based Microsystems'.

\section{References}

Adrus N and Ulbricht M (2012) Novel hydrogel pore-filled composite membranes with tunable and temperatureresponsive size-selectivity. Journal of Materials Chemistry 22: 3088-3098.

Arndt KF, Kuckling D and Richter A (2000) Application of sensitive hydrogels in flow control. Polymers for Advanced Technologies 11(8-12): 496-505.

Cervera J, Schiedt B and Ramírez P (2005) A PoissonNernst-Planck model for ionic transport through synthetic conical nanopores. Europhysics Letters 71(1): 35.

Chen D, Lear J and Eisenberg B (1997) Permeation through an open channel: Poisson-Nernst-Planck theory of a synthetic ionic channel. Biophysical Journal 72(1): 97.

Corry B, Kuyucak S and Chung S (2000a) Invalidity of continuum theories of electrolytes in nanopores. Chemical Physics Letters 320(1-2): 35-41.

Corry B, Kuyucak S and Chung SH (2000b) Tests of continuum theories as models of ion channels. ii. PoissonNernst-Planck theory versus Brownian dynamics. Biophysical Journal 78(5): 2364-2381.

Daiguji H (2010) Ion transport in nanofluidic channels. Chemical Society Reviews 39: 901-911.

Darvishmanesh S, Qian X and Wickramasinghe SR (2015) Responsive membranes for advanced separations. Current Opinion in Chemical Engineering 8: 98-104.

DeCoursey TE and Hosler J (2014) Philosophy of voltagegated proton channels. Journal of The Royal Society Interface 11(92): 20130799.

Ehrenhofer A, Bingel G, Paschew G, et al. (2016a) Permeation control in hydrogel-layered patterned PET membranes with defined switchable pore geometry - experiments and numerical simulation. Sensors and Actuators B: Chemical 232: 499-505.

Ehrenhofer A, Wallmersperger T and Richter A (2016b) Simulation of controllable permeation in PNIPAAm coated membranes. In: Proceedings of SPIE, behavior and 
mechanics of multifunctional materials and composites 2016, Las Vegas, Nevada, USA, 18 April 2016, volume 9800, p. 980016.

Frost S and Ulbricht M (2013) Thermoresponsive ultrafiltration membranes for the switchable permeation and fractionation of nanoparticles. Journal of Membrane Science 448: $1-11$.

Gerlach G and Arndt KF (2009) Hydrogel Sensors and Actuators: Engineering and Technology. Vol. 6. Berlin, Heidelberg: Springer Science \& Business Media.

Gouaux E and MacKinnon R (2005) Principles of selective ion transport in channels and pumps. Science 310(5753): 1461-1465.

Hirano-Iwata A, Niwano M and Sugawara M (2008) The design of molecular sensing interfaces with lipid-bilayer assemblies. TrAC Trends in Analytical Chemistry 27(6): $512-520$

Ito Y, Ochiai Y, Park YS, et al. (1997) pH-sensitive gating by conformational change of a polypeptide brush grafted onto a porous polymer membrane. Journal of the American Chemical Society 119(7): 1619-1623.

Keller K, Wallmersperger T, Kröplin B, et al. (2011) Modeling of temperature-sensitive polyelectrolyte gels by the use of the coupled chemo-electro-mechanical formulation. Mechanics of Advanced Materials and Structures 18(7): 511-523.

Kirby BJ (2010) Micro- and Nanoscale Fluid Mechanics: Transport in Microfluidic Devices. New York: Cambridge University Press.

Martinac B, Saimi Y and Kung C (2008) Ion channels in microbes. Physiological Reviews 88(4): 1449-1490.

Najem JS, Dunlap MD, Rowe ID, et al. (2015) Activation of bacterial channel MSCL in mechanically stimulated droplet interface bilayers. Scientific Reports 5: 1-11.

Nguyen J, Wei Y, Zheng Y, et al. (2015) On-chip sample preparation for complete blood count from raw blood. Lab Chip 15: 1533-1544.

Otto O, Rosendahl P, Mietke A, et al. (2015) Real-time deformability cytometry: On-the-fly cell mechanical phenotyping. Nature Methods 12(3): 199-202.
Park YS, Ito Y and Imanishi Y (1998) Permeation control through porous membranes immobilized with thermosensitive polymer. Langmuir 14(4): 910-914.

Paschew G, Schreiter J, Voigt A, et al. (2016) Autonomous chemical oscillator circuit based on bidirectional chemical-microfluidic coupling. Advanced Materials Technologies 1: 1600005.

Richter A, Kuckling D, Howitz S, et al. (2003) Electronically controllable microvalves based on smart hydrogels: Magnitudes and potential applications. Journal of Microelectromechanical Systems 12(5): 748-753.

Sarles SA and Leo DJ (2010) Regulated attachment method for reconstituting lipid bilayers of prescribed size within flexible substrates. Analytical Chemistry 82(3): 959-966.

Shields CW, Reyes CD and López GP (2015) Microfluidic cell sorting: A review of the advances in the separation of cells from debulking to rare cell isolation. Lab on a Chip 15(5): 1230-1249.

Singer SJ and Nicolson GL (1972) The fluid mosaic model of the structure of cell membranes. Science 175(4023): 720-731.

Squires TM and Quake SR (2005) Microfluidics: Fluid physics at the nanoliter scale. Reviews of Modern Physics 77(3): 977.

Tu B, Chen M, Xie Y, et al. (2013) A parallel finite element simulator for ion transport through three-dimensional ion channel systems. Journal of Computational Chemistry 34(24): 2065-2078.

Ulbricht M (2006) Advanced functional polymer membranes. Polymer 47(7): 2217-2262.

Zhang X, Xiang N, Tang W, et al. (2015) A passive flow regulator with low threshold pressure for high-throughput inertial isolation of microbeads. Lab on a Chip 15(17): 3473-3480.

Zheng Q, Chen D and Wei GW (2011) Second-order Poisson-Nernst-Planck solver for ion transport. Journal of Computational Physics 230(13): 5239-5262. 